\title{
Linear Dispersion Relation of Beta-Induced Alfvén Eigenmodes in Presence of Anisotropic Energetic Ions
}

\author{
Ruirui Ma, ${ }^{1}$ Ilija Chavdarovski, ${ }^{1}$ Gaoxiang Ye, ${ }^{2}$ and Xin Wang ${ }^{3, *}$ \\ ${ }^{1}$ Institute for Fusion Theory and Simulation and Department of Physics, \\ Zhejiang University, Hangzhou, China 310027 \\ ${ }^{2}$ Department of Physics, Zhejiang University, Hangzhou, China 310027 \\ ${ }^{3}$ Max-Planck-Institut für Plasmaphysik, \\ Boltzmannstr.2, 85748 Garching, Deutschland
}

(Dated: May 2, 2014)

\begin{abstract}
Using the theoretical framework of the generalized fishbone-like dispersion relation, the linear properties of beta-induced Alfvén eigenmodes (BAEs) and energetic particle continuum modes (EPMs) excited by anisotropic slowing-down energetic ions are investigated analytically and numerically. The resonant contribution of energetic ions to the potential energy perturbation as well as fluid-like term describing the background plasma and adiabatic contribution of energetic ions are derived. For high-mode numbers, numerical results show smooth transition between the EP continuous spectrum and BAEs in the gap. EPMs and/or BAEs are destabilized by energetic ions, with real frequencies and growth rates strongly dependendent on the energetic particle density and resonant frequency.
\end{abstract}

PACS numbers: 52.35.Bj; 52.55.Pi; 52.55.Tn

* corresponding author. Email address: xin.wang@ipp.mpg.de 


\section{INTRODUCTION}

Proper understanding of shear Alfvén wave (SAW) interaction with energetic particles (EPs) produced by auxiliary heating or nuclear fusion reactions in tokamaks is necessary for achieving better confinement of the latter, since resonant wave-particle interactions can destabilize Alfvén modes, which, conversely, can cause significant fast-ion redistribution or loss, and as a result substantial damage to the containment vessel [1-5]. Significant efforts, both experimental and theoretical, has been dedicted to this topic [2, 6-11]. Beta-induced Alfvén eigenmodes (BAEs) are particularly important since they can strongly interact with both thermal ions and EPs [5, 12-16].

Beta-induced Alfvén eigenmodes have typical frequencies located below the shear Alfvén continuous spectrum in a frequency gap caused by the finite thermal plasma compressibility $[5,17,18]$. Experimental evidence of BAE destabilization by energetic beam ions was first observed by Heidbrink on D-IIID tokamak [19]. Subsequently, BAEs have been observed in Ohmically heated plasmas in absence of energetic ions [20,21], as well as plasmas heated by ion cyclotron [16, 22] and electron cyclotron resonant heating [23]. Recent numerical simulations have also shown BAE excitation by EPs [24-26]. A general theoretical framework, the so-called generalized fishbone-like dispersion relation (GFLDR) [2, 5-7, 27-30], has been developed to describe the various Alfvénic fluctuations in tokamak plasmas observed in experiments and numerical simulations. EP continuum modes (EPMs), which are eigenmodes intrinsic to the presence of EPs and can't exist without a sufficient amount of EPs, have also been described by the GFLDR [2]. Using a general mode structure decomposition [31] and the WKB asymptotic matching method [8], the GFLDR can be expressed in a form of an energy functional

$$
D(\omega)=-i \Lambda(\omega)+\delta W_{f}+\delta W_{k}(\omega)=0
$$

where $\omega=\omega_{r}+i \gamma$ is the complex frequency of the mode, with $\omega_{r}$ and $\gamma$ the real frequency and growth/damping rate, respectively. $\Lambda$ represents the general inertia response and its form

depends on the relevant physics inside the inertial layer, while $\delta W_{f}$ and $\delta W_{k}$ are the fluid-like and the EP kinetic terms, respectively $[2,5,7] . \delta W_{f}$ is a real function of the equilibrium parameters, whereas $\delta W_{k}$ depends on $\omega$ and the characteristic frequencies of EP motion. The real part of $\delta W_{k}$ accounts for the non-resonant EP response, while the imaginary part comes from the resonant wave-particle interaction, and provides the instability mechanism 
necesary for the existence of EPMs, which are entirely determined by the EP characteristic quantities $[2,7]$.

The BAE dispersion relation is one limiting case of the GFLDR [5, 15, 28]. Aside from diamagnetic effects, kinetic descriptions of low frequency Alfvén modes have so far included wave-particle resonances with circulating thermal ions [5, 28] as well as deeply trapped ions and electrons [15, 32]. The simulations of BAEs excited by EPs have been limited to isotropic slowing down or Maxwellian distribution functions [24, 25]. However, EPs generated by neutral beam injection (NBI) heating are better described by anisotropic slowing down distribution function. In this paper, we analytically and numerically consider BAE and EPM excitation via transit resonance with hot ions generated by NBI heating in toroidal systems. Both the fluid-like and energetic particle terms are obtained analytically. Numerically solving the GFLDR we find that the EPMs and BAEs are destabilized by the circulating energetic ions via transit resonance for different sets of parameters: the energetic ion characteristic velocity $v_{E}$ and $\tau=T_{e} / T_{i}$ where $T_{e}$ and $T_{i}$ are electron and ion temperature in energy units, respectively, and there is a smooth transition from the continuum EPM spectrum to discrete BAE modes when these parameters are changing. Linear stabilities of $\mathrm{EPM}$ and BAE in the presence of anisotropic energetic ions are investigated, showing that for EPM the real frequency and growth rate increase with $\tau, v_{E}$ and energetic ion density $n_{E}$, with the latter one having a threshold for excitation of EPMs. For BAE the location of the real frequency inside the gap is determined by both the core plasma $\tau$ [5] and energetic ion (velocity and density). The growth rate of the nearly undamped BAE is mainly increasing with the energetic ion density or velocity, although some drive is necessary to overcome the finite Landau damping.

The paper is organized as follows: In Sec. II we present the BAE dispersion relation and the derivation of the relevant terms in the GFLDR. Numerical studies of the analytic dispersion relation of BAEs in the presence of moderate spatial gradients of the EPs with anisotropic slowing-down distribution are presented and discussed in Sec. III, with emphasis on the effects of EP density and velocity. We also give a brief discussion on the identification of continuum mode (EPM) vs. the discrete gap AE (BAE) in the light of the GFLDR. Conclusions are given in Sec. IV. 


\section{DISPERSION RELATION}

An equilibrium with shifted circular flux surfaces, large-aspect ratio $\epsilon=a / R_{0} \sim \mathcal{O}\left(10^{-1}\right)$ with $R_{0}$ and $a$ being the major and minor radii, respectively, and high- $\beta\left(=8 \pi P / B^{2} \approx \epsilon\right.$ where $P$ is the plasma pressure and $B$ the equilibrium magnetic field) is adopted. The equilibrium is entirely determined by a set of parameters $(s-\alpha)$ [33], namely the average magnetic shear $s=r q^{\prime} / q$, and the normalized pressure gradient $\alpha \equiv-R_{0} q^{2} \beta^{\prime}$, where $q$ is the safety factor, $r$ is the radial coordinate and the prime denotes derivation with respect to $r$. We assume the plasma consists of two components, a relatively cold fluid-like background plasma with isotropic Maxwellian particle distribution, and an EP component with anisotropic slowing down distribution. For the modes of interest in this paper we adopt the following frequency $\omega$ and wavelength $k$ orderings [7]: $\omega \approx \omega_{* p i} \approx \omega_{t i} \approx \mathcal{O}\left(\epsilon^{1 / 2}\right) \omega_{A}\left(\omega_{A}=v_{A} / q R_{0}\right), k_{\vartheta} \rho_{L i} \approx \mathcal{O}(\epsilon)$, $k_{\vartheta} \rho_{L E} \sim \mathcal{O}\left(\epsilon^{1 / 2}\right)$. Here, $k_{\vartheta}$ is the wave vector in the poloidal direction, $\rho_{L i}$ and $\rho_{L E}$ the thermal and energetic ion Larmor radii, respectively, $\omega_{* p i}=\left(c T_{i} / e_{i} B^{2}\right)(\mathbf{k} \times \mathbf{B}) \cdot \nabla \ln P_{i}$ is the thermal ion diamagnetic frequency, $\omega_{t i}=\sqrt{2 T_{i} / m_{i}} / q R_{0}$ the thermal ion transit frequency, $e_{i}$ and $m_{i}$ the ion electric charge and mass, respectively, $P_{i}$ the thermal ion pressure and $\mathbf{k}$ the wavevector.

Within the present model, the plasma is described by two variables [5]: the perturbed scalar potential $\delta \phi$ and the vector potential $\delta \psi$, related to the perpendicular magnetic field as $\delta A_{\|} \equiv-i\left(\frac{c}{\omega}\right) \mathbf{b} \cdot \nabla \delta \psi$ where $\mathbf{b}=\mathbf{B} / B$. Note that the modes of interest here are characterized by the SA time scale which is much longer than that of the compressional Alfvén wave. Therefore, we have the perpendicular press balance, i.e., $\nabla_{\perp}\left(B \delta B_{\|}+4 \pi \delta p_{\perp}\right)=0[7,34,35]$. Standard procedure $[5,36]$, beginning with the gyrokinetic and quasi-neutrality equations and adopting the ballooning mode formulation, leads to the so-called vorticity equation in terms of the extended poloidal angle $\theta[37]$

$$
\left[\frac{\partial^{2}}{\partial \theta^{2}}+\Lambda^{2}+\frac{\alpha \cos \theta}{f}-\frac{(s-\alpha \cos \theta)^{2}}{f^{2}}\right] \delta \Psi-f^{-1 / 2}\left\langle\frac{4 \pi e_{E} q^{2} R_{0}^{2}}{k_{\vartheta}^{2} c^{2}} J_{0}\left(\lambda_{\rho_{E}}\right) \omega \omega_{d E} \delta K_{E}\right\rangle v=0
$$

where $\delta \Psi=f^{1 / 2} \delta \psi, f=1+(s \theta-\alpha \sin \theta)^{2}$ and we have assumed the validity of ideal MHD approximation $\delta \phi=\delta \psi[2,5,7]$. In Eq. (2), $\omega_{d E}$ is the magnetic drift frequency of EP with $\omega_{d E}=k_{\vartheta} \Omega_{d E} g(\theta), \Omega_{d E}=\left(v_{\perp}^{2} / 2+v_{\|}^{2}\right)_{E} / \omega_{c E} R_{0}, g(\theta)=\cos \theta+(s \theta-\alpha \sin \theta) \sin \theta, J_{0}$ is the

Bessel function of the first kind and zero index, with argument $\lambda_{\rho_{E}}=k_{\perp} \rho_{L E}, k_{\perp}^{2}=k_{\vartheta}^{2}+k_{r}^{2}$, $\rho_{L E}=v_{\perp E} / \omega_{c E}, \omega_{c E}=q_{E} B / m_{E} c, q_{E}$ and $m_{E}$ are the electric charge and mass of the 
energetic ions, $\langle\ldots\rangle_{v}=2 \pi \sum_{\sigma= \pm 1} \int d \varepsilon d \mu B /\left|v_{\|}\right|$with $\sigma=v_{\|} /\left|v_{\|}\right|, \varepsilon=v^{2} / 2, \mu=v_{\perp}^{2} / 2 B$, the subscripts $\|$ and $\perp$ represent parallel and perpendicular components referred to the direction of the equilibrium magnetic field $\mathbf{b}$. Compressional effects from both the core and energetic components are included in the above equation, while for the expression of $\Lambda$ we use the form derived in Ref. 5, in which both thermal ion transit resonances as well as diamagnetic effects (finite $\omega_{* p i}$ ) are maintained. The non-adiabatic EP response $\delta K_{E}$ is governed by the following gyrokinetic equation [34, 38, 39]

$$
\left[\omega_{t E} \frac{\partial}{\partial \theta}-i\left(\omega-\omega_{d E}\right)\right] \delta K_{E}=i \frac{e_{E}}{m_{E}} Q F_{0 E} \frac{\omega_{d E}}{\omega} J_{0}\left(\lambda_{\rho_{E}}\right) f^{-1 / 2} \delta \Psi
$$

where $\omega_{t E}=v_{\| E} / q R_{0}, Q F_{0 E}=\left(\omega \partial_{\varepsilon}+\hat{\omega}_{* E}\right) F_{0 E}, \hat{\omega}_{* E} F_{0 E}=\omega_{c E}^{-1}(\mathbf{k} \times \mathbf{b}) \cdot \nabla F_{0 E}$ and $F_{0 E}$ is the EP equilibrium distribution function.

Equation (2) is obtained in the long wavelength limit $k_{\vartheta} \rho_{L i} \ll 1$, assuming adiabatic electron response $\left(\delta K_{e}=0\right)$. We treat EPs dynamics nonperturbatively and consider the finite orbit size effects. We also neglect EPs density $n_{E}$, but keep its pressure $P_{E}$, which is comparable to that of the thermal ion, consistently with a nonperturbative approach. Note that the MHD adiabatic EP compression enters Eq. (2) via the equilibrium parameter $\alpha$, whereas the kinetic non-adiabatic EP compression (last term in Eq. (2)) contributes to the vorticity equation by coupling the pressure perturbation via the magnetic curvature drift [36].

Equation (2) exhibits two-scale-length structure, i.e., the function $\delta \Psi$ varies on a small angle $\theta_{0} \approx 1$ and a large angle $\theta_{1} \approx 1 / \beta^{1 / 2}[5]$. For $k_{\vartheta} \rho_{L E} \lesssim 1$, because of orbit averaging, the finite Larmor radius (FLR) and finite orbit width (FOW) effects of the energetic ions are negligible in the inertial layer $\theta \approx \theta_{1}$, whereas the same contribution can be finite in the ideal region $\theta \approx \theta_{0}$. The local BAE dispersion relation in the presence of energetic ions (Eq. (1)) is derived by asymptotically matching the solutions of the eigenmode Eq. (2) in the inertial layer and the ideal region [5].

\section{A. The energetic ion term $\delta W_{k}$}

The total contribution of the EPs to the GFLDR can be written as $\delta W_{E}(\omega)=\delta W_{f, E}+$ $\delta W_{k}(\omega)$, where the $\delta W_{f, E}$ term represents the adiabatic and convective responses [6] which enters the usual fluid-like term $\delta W_{f}$ in the form of $\xi \cdot \nabla P_{E}$, with $\xi$ being the plasma dis- 
placement. On the other hand, $\delta W_{k}$ is the nonadiabatic contribution and for circulating particles is given by [7]

$$
\delta W_{k u} \simeq \frac{\pi^{2} q_{E}^{2} q^{2} R_{0}^{2}}{2 m_{E} c^{2} s}\left\langle\frac{Q F_{0 E} \Omega_{d E}^{2}}{\Delta\left(1+\Delta^{2}\right)^{3 / 2}} \frac{\omega}{\omega_{t E}^{2}-\omega^{2}}\right\rangle_{v},
$$

where $\Delta^{2} \equiv k_{\vartheta}^{2}\left(\rho_{L E}^{2}+\rho_{d E}^{2} / 2\right) / 4$ contains the FLR and FOW $\left(\rho_{d E}=\Omega_{d E} / \omega_{t E}\right)$ effects of the EP. In the present work we mainly consider $\triangle \ll 1$, since it is the most unstable wave vector range $[2,7]$.

In order to model the NBI generated energetic ions we choose a symmetric in $v_{\|}$slowingdown beam ion distribution function, with a single pitch angle $(\lambda=\mu / \varepsilon)$

$$
F_{0 E}=\frac{\sqrt{2\left(1-\lambda_{0} B_{0}\right)} B_{0} \beta_{E}(r)}{2^{5} \pi^{2} m_{E}\left(\varepsilon_{b}-\varepsilon_{c}\right)} \frac{\delta\left(\lambda-\lambda_{0}\right)}{\varepsilon_{c}^{3 / 2}+\varepsilon^{3 / 2}},
$$

where $\beta_{E} \equiv 2 \mu_{0} P_{E} / B_{0}^{2}, \delta(x)$ is the Dirac function, $\lambda_{0}$ is the energetic ion birth pitch angle, $\varepsilon \in\left[\varepsilon_{c}, \varepsilon_{b}\right], \varepsilon_{b}$ and $\varepsilon_{c}$ are the birth (maximum) and critical energies of EPs, respectively. For burning plasmas $\varepsilon_{c} \ll \varepsilon_{b}$ [40], but we have kept $\varepsilon_{c}$ in Eq. (5) for consistency with models often adopted by numerical codes; e.g., [41, 42].

Substituting Eq. (5) into Eq. (4) we obtain

$$
\delta W_{k u}=\frac{\pi \alpha_{E}\left(1-\lambda_{0} B_{0} / 2\right)}{2 \sqrt{2} s\left(1-1 / N_{b c}\right)} \cdot \bar{\omega}\left[2\left(1-1 / \sqrt{N_{b c}}\right)-\bar{\omega} \ln \left(\frac{\bar{\omega}+1}{\bar{\omega}-1}\right)+\bar{\omega} \ln \left(\frac{\sqrt{N_{b c}} \bar{\omega}+1}{\sqrt{N_{b c}} \bar{\omega}-1}\right)\right]
$$

where $\alpha_{E} \equiv q^{2} R_{0} \beta_{E}^{\prime}, \bar{\omega}=\omega / \omega_{t m}$ with $\omega_{t m}$ being the transit frequency at the maximum particle energy, and $N_{b c}=\varepsilon_{b} / \varepsilon_{c}$. Here, we have neglected the velocity space damping due to energetic ions and considered that the EP drive comes mainly from the pressure gradient in the real space, i.e., $\hat{\omega}_{* E} \gg \omega$. Furthermore, only the EP FOW effect is kept since $\rho_{d E} \gg \rho_{L E}$.

Equation (6) implies that resonance occurs when the frequency of the mode is close to the transit frequency of the EPs, and the source of the instability is related to radial pressure gradient contained in $\alpha_{E}$.

\section{B. The fluid-like term $\delta W_{f}$}

The expression of $\delta W_{f}$ is given in Refs. 2, 5, and 7 for thermal plasma component. This term, however, should include both core-plasma $\delta W_{f c}$ and EP pressure contribution $\delta W_{f E}$ in the presence of energetic ions. The MHD non-adiabatic particle compression (MPC) term, 
contributing to $\delta W_{f}$, can be expressed as [36]

$$
\mathrm{MPC}=\frac{4 \pi}{B^{2} k_{\vartheta}^{2}} \sum_{s=i, E}\left\langle\mathbf{k} \times \mathbf{b} \cdot \tilde{\nabla}\left(P_{s \|}+P_{s \perp}\right) \Omega_{\kappa} \delta \psi\right\rangle_{s} .
$$

For thermal plasma with isotropic Maxwellian distribution we assume $P_{i \|}=P_{i \perp}$, while for passing EPs created by parallel neutral beam injection, $P_{E \|} \gg P_{E \perp}$ and $\eta_{E}=$ $\partial \ln T_{E} / \partial \ln n_{E}=0$, i.e., the temperature of energetic ions is flat. Transforming Eq. (7) to the ballooning space, the MPC term can now be written in a more convenient form: $\alpha g(\theta) / q^{2} R_{0}^{2}$, where $\alpha=\alpha_{c}+\alpha_{E}, \alpha_{c}=(1+\tau)\left(1+\eta_{i}\right) q^{2} \beta_{i} / \epsilon_{n i}$ for the core plasma and $\alpha_{E}=q^{2} \beta_{E} / \epsilon_{n E}$ for energetic ions. Here, $\eta_{i}=\partial \ln T_{i} / \partial \ln n_{i}, \epsilon_{n i}=L_{n i} / R_{0}$, and $\epsilon_{n E}=L_{n E} / R_{0}$ with $L_{n i}$ and $L_{n E}$ the density gradient scale lengths of the thermal and energetic ions, respectively.

The solution for the eigenfunction $\delta \Psi$ in the ideal region has to asymptotically match the inertial layer solution $\delta \Psi \sim e^{i \Lambda|\theta|}$. The expression for the fluid-like term is given by [7]

$$
\delta W_{f}=\frac{1}{2} \int_{-\infty}^{\infty} d \theta\left\{\left|\frac{d \delta \Psi}{d \theta}\right|^{2}+\left[\frac{(s-\alpha \cos \theta)^{2}}{f^{2}}-\frac{\alpha \cos \theta}{f}\right]|\delta \Psi|^{2}\right\} .
$$

If $|s| \sim \alpha<1$, it can be shown that high accuracy can be achieved with the trial function $\delta \Psi=1+\alpha \cos \theta / f$ based on asymptotic variational method [43]. Straight forward algebra yields

$$
\delta W_{f} \simeq \frac{\pi}{|s|}\left[\frac{s^{2}}{4}-\frac{3 \alpha^{2}|s|}{2}+\frac{5 \alpha^{2} s^{2}}{32}+\frac{45 \alpha^{4}}{128}-\left(1+\frac{\alpha}{2}\right) e^{-1 /|s|}\right],
$$

where in the integration we have adopted $f \approx 1+s^{2} \theta^{2}$ and neglected the oscillatory terms

$\propto \cos n \theta$ with $n=2$ or 3 . The term proportional to $e^{-1 /|s|} \operatorname{comes}$ from $\cos \theta$ related term and is a result of overlapping scales $\theta \sim 1 /|s|$ and $\theta \sim 1$. This term is accurate near the first stability region, where $|s| \sim \alpha^{2}$ for small $|s|$ and $\alpha$. The leading order terms in Eq. (9) are very close to those obtained in Ref. 44.

\section{NUMERICAL RESULTS}

We numerically solve the linear dispersion relation Eq. (1), where $\delta W_{k}$ and $\delta W_{f}$ are given by Eqs. (6) and (9), respectively, while for $\Lambda$ we use Eq. (19) of Ref. 5. Based on Eq. (1), we identify two types of modes: EP continuum modes with $\operatorname{Re}\left(\Lambda^{2}\right)>0$ and discrete gap modes with $\operatorname{Re}\left(\Lambda^{2}\right)<0$. The EP continuum modes experience strong continuum damping 

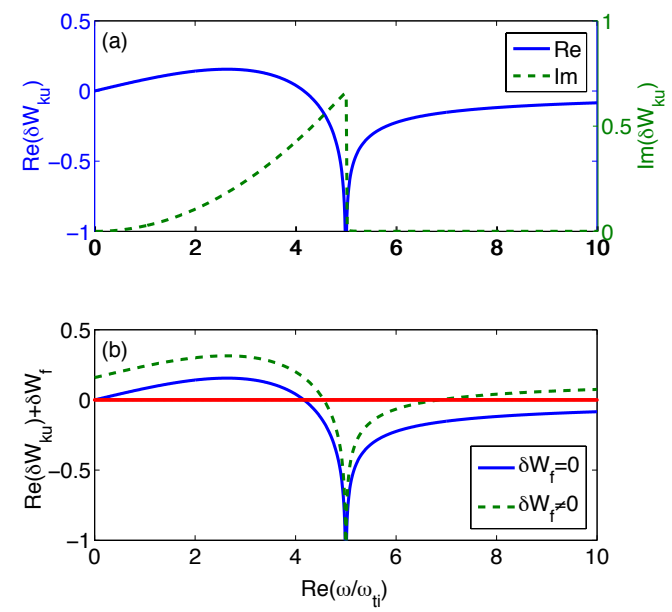

FIG. 1. (a) Real (solid line) and imaginary (dashed line) values of $\delta W_{k u}$ as a function of $\operatorname{Re}\left(\omega / \omega_{t i}\right)$. (b) Values of $\operatorname{Re}\left(\delta W_{k u}\right)+\delta W_{f}$ are shown as a function of $\operatorname{Re}\left(\omega / \omega_{t i}\right)$. Here, $\operatorname{Im}\left(\omega / \omega_{t i}\right)=0.001$, $v_{E i}=5.0, n_{E i}=0.01$, and $\tau=1$.

and require suffiently strong EP drive as threshold excitation condition. On the other hand, the gap modes are weakly coupled to the continuum and only require a small drive from the energetic particles and/or thermal ions to compensate Landau damping [14, 27]. Since the SAW accumulation point is given by $\Lambda^{2}=0$, the transition between the continuum and the gap modes occurs around $\operatorname{Re}\left(\delta W_{k u}\right)+\delta W_{f} \simeq 0$. The real frequency of the EPM is located within the shear Alfvén continuum spectrum, while the growth rate is given by [7]

$$
\operatorname{Im}\left(\omega / \omega_{t i}\right) \simeq \frac{\operatorname{Im}\left(\delta W_{k u}\right)-\operatorname{Re} \Lambda}{-\partial \operatorname{Re}\left(\delta W_{k u}\right) / \partial \operatorname{Re}\left(\omega / \omega_{t i}\right)},
$$

where $\operatorname{Im}\left(\delta W_{k u}\right)$ describes the energetic particle drive and $\operatorname{Re} \Lambda$ the continuum damping. The threshold condition is thus $\operatorname{Im}\left(\omega / \omega_{t i}\right) \geq 0$ for $\operatorname{Im}\left(\delta W_{k u}\right) \geq \operatorname{Re} \Lambda$ and $\partial \operatorname{Re}\left(\delta W_{k u}\right) / \partial \operatorname{Re}\left(\omega / \omega_{t i}\right)<$ $0[2,5,7,30,45]$. For the BAE the condition $\operatorname{Re}\left(\delta W_{k u}\right)+\delta W_{f}<0$ allows for the mode to be localized inside the gap. $\operatorname{Re}\left(\delta W_{k u}\right)$ provides a real frequency shift, i.e., it removes the degeneracy with the continuum accumulation point, while $\operatorname{Im}\left(\delta W_{k u}\right)$ privides the mode drive and compensates for the small, but non-negligible damping from the thermal ions [27, 36].

Further in this section, we use the following values for the local equilibrium parameters $\beta_{i}=0.01, q=2.0, s=0.25, \epsilon=0.3, \omega_{* n i} / \omega_{t i}=0.1, \eta_{i}=2.0, \eta_{E}=0.0, \lambda_{0} B_{0}=0.0$, $N_{b c}=10^{2}, L_{n i} / R_{0}=3.0$, and $L_{n E} / R_{0}=0.15$. We assume large ratio of beam to thermal ion transit frequency $\omega_{t E} / \omega_{t i}=v_{E} / v_{i} \equiv v_{E i}=5.0$ in order to correctly estimate the importance of the transit resonance. In Fig. 1(a), we show the value of $\operatorname{Re}\left(\delta W_{k u}\right)$ and $\operatorname{Im}\left(\delta W_{k u}\right)$ as 

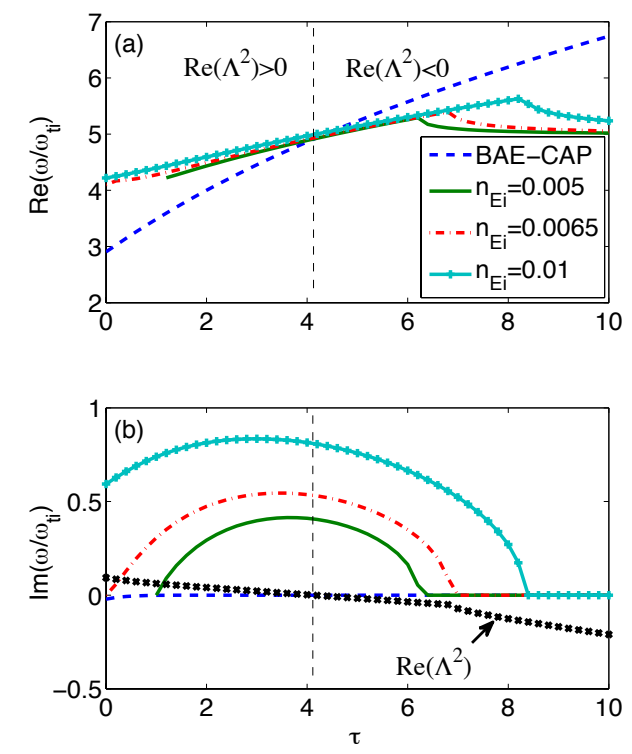

FIG. 2. Frequencies (a) and growth rates (b) vs. $\tau$ for $\Lambda^{2}=0$ (BAE-CAP), $n_{E i}=0.005$, $n_{E i}=0.0065$, and $n_{E i}=0.01$. The line with crosses shows $\operatorname{Re}\left(\Lambda^{2}\right)$ vs. $\tau$ for fixed $n_{E i}=0.0065$. Here, $\delta W_{f}=0, \beta_{i}=0.01, q=2.0, s=0.25, \epsilon=0.3, \omega_{* n i} / \omega_{t i}=0.1, \eta_{i}=2.0, \eta_{E}=0.0, \lambda_{0} B_{0}=0.0$, $N_{b c}=10^{2}, L_{n i} / R_{0}=3.0, L_{n E} / R_{0}=0.15$, and $v_{E i}=5.0$.

functions of real mode frequency. The imaginary part of $\delta W_{k u}$ is positive with $\omega \leq \omega_{t m}$, while the real part is positive for small values of $\omega / \omega_{t i}$, but changes sign at frequency below the resonant energetic ion frequency, as does $\operatorname{Re}\left(\delta W_{k u}\right)+\delta W_{f}$ in Fig. 1(b). In the same figure the solution of $\operatorname{Re}\left(\delta W_{k u}\right)+\delta W_{f}=0$ determines the location of the transition point between EPM and BAE. We can see $\delta W_{f} \neq 0$ can affect the real frequency of the EPM.

The transition between EPM and BAE spectrum is shown in Fig. 2 for increasing $\tau$ from 0 to 10 . The SAW continuum accumulation point $(\mathrm{CAP})\left(\Lambda^{2}=0\right)$ is given by the blue dashed line; and its dependence on $\tau$ [5] illustrates why this is a good parametric scan to perform to investigate the smooth transition from EPM to BAE. For $\tau=0$ the EP transit frequency drives the mode within the shear Alfvén continuous spectrum, while for $\tau=10$ the mode is most efficiently driven in the BAE frequency gap. The other three curves characterize different solutions of the GFLDR for different values of the normalized density, $n_{E i} \equiv n_{E} / n_{i}$ of energetic ions. The four lines intersect around the same point $\operatorname{Re}\left(\omega / \omega_{t i}\right) \simeq 5.0$, which can be taken to be the transition between the EPM region $\left(\operatorname{Re}\left(\Lambda^{2}\right)>0\right)$ with frequencies above the accumulation point and the BAE region $\left(\operatorname{Re}\left(\Lambda^{2}\right)<0\right)$ with frequencies below the 

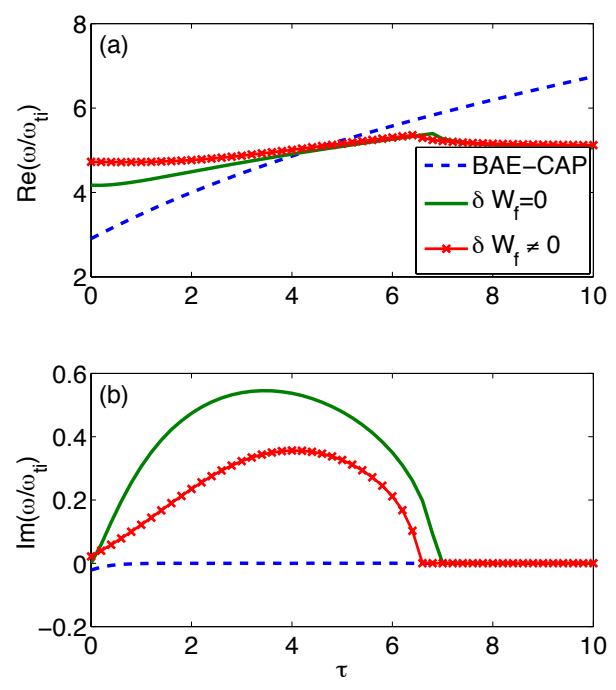

FIG. 3. Real frequencies and growth rates vs. $\tau$ for $\Lambda^{2}=0$ (BAE-CAP), without $\delta W_{f}$, and with $\delta W_{f}$. Here, $n_{E i}=0.0065$ and $v_{E i}=5.0$. The other parameters are the same as in Fig. 2.

CAP. This classification is strictly valid near marginal stability $\gamma \ll \omega_{r}$ that, given Fig. 2, is a reasonable assumption. For EPM the frequency (Fig. 2(a)) as well as the corresponding growth rate (Fig. 2(b)) increase with $\tau$ in all the three cases, due to the dependence on $\tau$ of the CAP; but unlike the real frequency the growth rates are strongly dependent on the EP density $n_{E i}$. It is worth noting that the energetic particle drive becomes stronger with increasing $n_{E i}$, since $\operatorname{Im}\left(\delta W_{k u}\right) \propto \alpha_{E}$ (see Eq. (6)), and Eq. (10) implies there is density threshold for the excitation of EPM [7]. Figure 2 shows for $\tau=1$ EPM cannot exist below $n_{E i}=0.005$ which is the threshold density for EPM excitation. For BAE the frequencies initially increase to certain maximal values and then decrease with $\tau$, while the corresponding growth rates decrease to zero, consistent with the analyses of Ref. [5]. We can understand these results by comparing Fig. 1(a) where the particle drive is strongest around the resonance frequency that is also the transition between EPM and BAE, with Fig. 2(b). Above this frequency the drive is weak and the mode eventually becomes marginally stable.

In Fig. 3 we show $\operatorname{Re}\left(\omega / \omega_{t i}\right)$ and $\operatorname{Im}\left(\omega / \omega_{t i}\right)$ versus $\tau$ with and without $\delta W_{f}$ in order to highlight the effect of the fluid-like term $\delta W_{f}$ on the GFLDR. The blue dashed line represents the BAE CAP. The green solid line gives the frequency (Fig. 3(a)) and growth rate (Fig. 3(b)) of the modes without the fluid-like term, while the red line with crosses is 

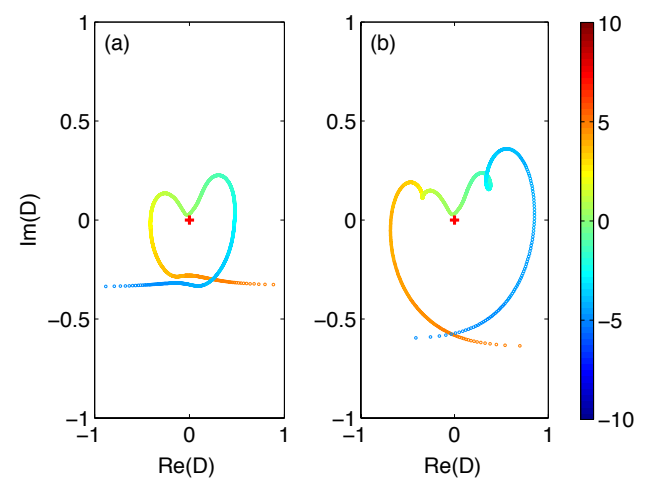

FIG. 4. The complex $\mathrm{D}(\omega)$ plane for the single-pitch angle slowing-down distribution showing the Nyquist plots for $n_{E i}=0.01$ (a) $\tau=1$ (EPM) and (b) $\tau=4$ (BAE). Here, $v_{E i}=5.0$. The other parameters are the same as in Fig. 2.

for $\delta W_{f} \neq 0$. When $\delta W_{f}>0$, the growth rates of the modes are decreased, which is to be expected since $\delta W_{f}>0$ implies stabilizing MHD effects. We also find that the transition point between the EPM and BAE spectrum is shifted slightly up, which can be explained by Fig. 1(b) when $\delta W_{f}$ is included.

Depending on the value of $\operatorname{Re}\left(\delta W_{k u}\right)+\delta W_{f}$ we obtain either EPM or gap mode, only one of which can be excited for a given set of parameters. To demonstrate this, we show the Nyquist diagrams for expression $\operatorname{Re}(D(\omega))$ with $D(\omega)=i \Lambda-\delta W_{k u}=0$, where for simplicity we have taken $\delta W_{f}=0$. As shown in Fig. 4(a) for the EPM and Fig. 4(b) for BAE region, the path in the $D$-plane encircles the origin only once, thus confirming there is only one unstable mode in each case. With the aid of the Nyquist analysis, spurious nonzero solutions produced by singularities of the transcendental function can be excluded.

The BAE and EPM frequencies and growth rates as functions of the energetic ion velocity $v_{E i}$ and density $n_{E i}$ are presented in Figs. 5(a) and (b), respectively. The parameters in Fig. $5(\mathrm{a}), \tau=5$ and $n_{E i}=0.01$ are kept fixed. In Fig. 5(b) for the BAE case (the thin lines) we set $\tau=1$ and $v_{E i}=3.0$, while for the EPM case (the thick lines) $\tau=1$ and $v_{E i}=4.0$. The red dot lines represent the CAP of BAE for $\tau=5$ (Fig. 5(a)) and $\tau=1$ (Fig. 5(b)), respectively. The mode below the CAP is BAE, while the one above is EPM. In Fig. 5(a), the BAE real frequency decreases with $v_{E i}$ in the region $v_{E i} \lesssim 4.3$, while the growth rate is zero, i.e., the BAE is marginally stable. Above $v_{E i} \gtrsim 4.3$ the BAE is driven unstable by the energetic ions and both the growth rate and real frequency increase with $v_{E i}$. For 


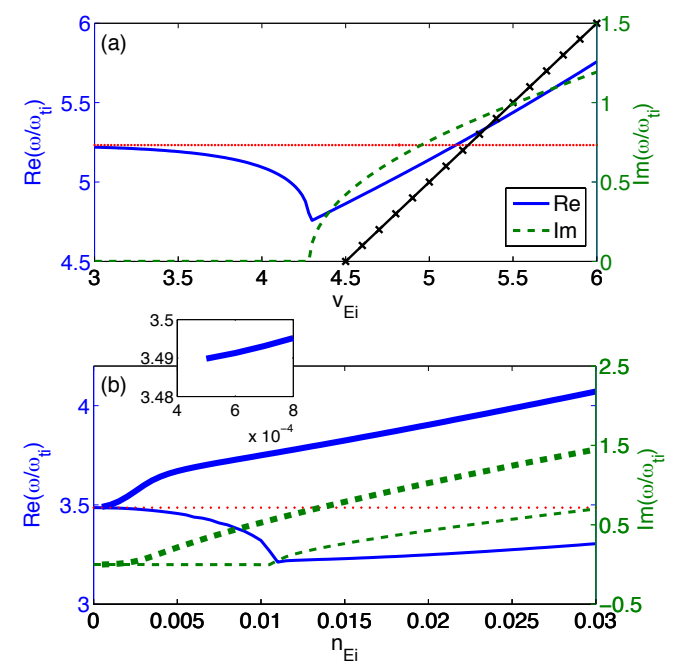

FIG. 5. Frequency (solid line) and growth rate (dashed line) of the mode vs. (a) $v_{E i}\left(n_{E i}=\right.$ $0.01, \tau=5)$ (The solid line with crosses shows the EP transit frequencies.) and (b) $n_{E i}(\tau=1$, $v_{E i}=3.0$ for BAE (thin lines) and $\tau=1, v_{E i}=4.0$ for EPM (thick lines)). Inset is an enlarged view of the EPM at small $n_{E i}$. Here, $\delta W_{f}=0$. The other parameters are the same as in Fig. 2 .

EPM above the CAP, the frequency increases with $v_{E i}$ and remains near the resonance with the energetic ions. The growth rate also increases, reflecting the $\omega / \omega_{t E}$ dependence in Eqs. (6) and (10). The inset, an enlarged view of the small $n_{E i}$ region, demostrates that EPM does not exist for $n_{E i} \leq 0.0005$, which is the threshold EP density for the parameters $\tau=1$ and $v_{E i}=4.0$. The mode frequency slightly increases towards the resonant frequency with increasing density $n_{E i}$, whereas the growth rate increases greatly. For BAE the tendency is the same as for changing $v_{E i}$ in Fig. 5(a).

\section{CONCLUSION}

Numerical analysis of the generalized fishbone-like dispersion relation has been performed in order to investigate the stability properties of EPMs and BAEs excited by passing energetic ions produced by NBI heating in tokamak plasmas. It's shown that there is a smooth transition between the EPM and BAE spectrum, described by the generalized fishbone-like dispersion relation. Both EPMs and BAEs can be linearly excited by energetic ions under proper choice of equilibruim profiles (background plasma and energetic ion parameters), 
which generally allow only one type of mode (BAE or EPM) to exist. The wave-particle resonant interaction with energetic ions is essential for the excitation of the mode in the long wavelength limit considered here. The self-consistent fluid-like $\delta W_{f}$ term has important effects on the dispersion relation and it exerts a significant stabilizing influence on the low frequency modes. Finally, the BAE and EPM frequencies are always closely related to the resonant hot ion frequency, with the growth rates increasing as $v_{E i}$ or $n_{E i}$ increase.

It should be pointed out that the present analysis is only local [5]. A global stability analysis, involving WKB radial mode structure effects from finite $\theta_{k} \equiv\left(n q^{\prime}\right)^{-1} k_{r}$, modifies the GFLDR [29, 30] and will be considered in a separated paper. Furthermore, the $\Lambda$ term in the BAE dispersion relation does not include the deeply [15] and barely trapped particle dynamics, nor finite Larmor radius and finite magnetic-orbit width effects [14, 46]. This, however, is consistent with the long wavelength limit considered here. Thus, our analytical dispersion relation for the BAEs excitation accounts for the essential physics in a transparent manner. An extension of the present analysis to shorter wavelengths, would deal with the couplong of BAE and KBM branches [5, 15, 29, 30, 32] and the excitation of Alfvén ion temperature gradient (AITG) driven modes [14], which is beyond the scope of this investigation.

\section{ACKNOWLEDGMENTS}

The authors would like to acknowledge useful discussions with L. Chen, F. Zonca, Z. Y. Qiu, H. S. Xie, L. Yang, and J. Zhu. This work is supported by the ITER-CN (no. 2013GB104004; 2013GB111004), NNSFC (no. 11105119; 11074215; 11374082) and the Key Laboratory of Basic Plasma Physics, Chinese Academy of Science (CAS).

\section{REFERENCES}

[1] D. J. Sigmar, C. T. Hsu, R. White, and C. Z. Cheng, Phys. Fluids B 4, 1506 (1992).

[2] L. Chen, Phys. Plasmas 1, 1519 (1994).

[3] R. A. Santoro and L. Chen, Phys. Plasmas 3, 2349 (1996). 
[4] L. Chen and F. Zonca, Physica Scripta T60, 81 (1995).

[5] F. Zonca, L. Chen, and R. A. Santoro, Plasma Phys. Control. Fusion 38, 2011 (1996).

[6] L. Chen and R. B. White, Phys. Rev. Lett. 52, 1122 (1984).

[7] S. Tsai and L. Chen, Phys. Fluids B 5, 3284 (1993).

[8] F. Zonca and L. Chen, Phys. Rev. Lett. 68, 592 (1992).

[9] G. Y. Fu, R. Nazikian, R. Budny, and Z. Chang, Phys. Plasmas 5, 4284 (1998).

[10] F. Zonca and L. Chen, Phys. Plasmas 7, 4600 (2000).

[11] W. W. Heidbrink, Phys. Plasmas 15, 055501 (2008).

[12] F. Zonca, L. Chen, R. A. Santoro, and J. Q. Dong, Plasma Phys. Control. Fusion 40, 2009 (1998).

[13] J. Dong, L. Chen, and F. Zonca, Nucl. FusionNucl. Fusion 39, 1041 (1999).

[14] F. Zonca, L. Chen, J. Q. Dong, and R. A. Santoro, Phys. Plasmas 6, 1917 (1999).

[15] I. Chavdarovski and F. Zonca, Plasma Phys. Control. Fusion 51, 115001 (2009).

[16] C. Nguyen, X. Garbet, R. Sabot, L.-G. Eriksson, M. Goniche, P. Maget, V. Basiuk, J. Decker, D. Elbeze, G. T. A. Huysmans, A. Macor, J.-L. Segui1, and M. Schneider, Plasma Phys. Control. Fusion 51, 095002 (2009).

[17] M. S. Chu, J. M. Greene, L. L. Lao, A. D. Turnbull, and M. S. Chance, Phys. Fluids B 4, 3713 (1992).

[18] A. D. Turnbull, E. J. Strait, W. W. Heidbrink, M. S. Chu, H. H. Duong, J. W. Greene, L. L. Lao, T. S. Taylor, and S. J. Thompson, Phys. Fluids B 5, 2546 (1993).

[19] W. W. Heidbrink, E. J. Strait, M. S. Chu, and A. D. Turnbull, Phys. Rev. Lett 71, 855 (1993).

[20] P. Buratti, P. Smeulders, F. Zonca, S. Annibaldi, M. D. Benedetti, H. Kroegler, G. Regnoli, O. Tudisco, and the FTU-team, Nucl. Fusion 45, 1446 (2005).

[21] S. V. Annibaldi, F. Zonca, and P. Buratti, Plasma Phys. Control. Fusion 49, 475 (2007).

[22] P. Lauber, M. Brudgam, D. Curran, V. Igochine, K. Sassenberg, S. Gunter, M. Maraschek, M. Garcia-Munoz, N. Hicks, and the ASDEX Upgrade Team, Plasma Phys. Control. Fusion 51, 124009 (2009).

[23] W. Chen, X. T. Ding, Y. Liu, Q. W. Yang, X. Q. Ji, Y. P. Zhang, Y. B. Dong, Y. Huang, J. Zhou, Y. Zhou, W. Li, B. B. Feng, X. M. Song, J. Q. Dong, Z. B. Shi, X. R. Duan, and the HL-2A team, Nucl. Fusion 51, 063010 (2011). 
[24] X. Wang, F. Zonca, and L. Chen, Plasma Phys. Control. Fusion 52, 115005 (2010).

[25] H. Zhang, Z. Lin, I. Holod, X. Wang, Y. Xiao, and W. Zhang, Phy. Plasmas 17, 112505 (2010).

[26] L. Qi, J. Q. D. A. Bierwage, G. Lu, and Z. M. Sheng, Phys. Plasmas 20, 032505 (2013).

[27] L. Chen and F. Zonca, Nucl. Fusion 47, S727 (2007).

[28] F. Zonca, A. Biancalani, I. Chavdarovski, L. Chen, C. D. Troia, and X. Wang, Journal of Physics: Conference Series 260, 012022 (2010).

[29] L. Chen and F. Zonca, Rev. Mod. Phys., submitted (2014).

[30] F. Zonca and L. Chen, Phys. Plasmas, submitted (2014).

[31] F. Zonca, R. B. White, and L. Chen, Phys. Plasmas 11, 2488 (2004).

[32] I. Chavdarovski and F. Zonca, Phys. Plasmas, submitted (2014).

[33] J. W. Connor, R. J. Hastie, and J. B. Taylor, Phys. Rev. Lett. 40, 6 (1978).

[34] L. Chen and A. Hasegawa, J. Geophys. Res. 96, 1503 (1991).

[35] F. Zonca and L. Chen, Phys. Fluids B 5, 3668 (1993).

[36] F. Zonca and L. Chen, Plasma Phys. Control. Fusion 48, 537 (2006).

[37] J. Connor, R. Hastie, and J. Taylor, Proc. R. Soc. London, Ser. A. 365, 1 (1979).

[38] T. M. Antonsen, Jr., and B. Lane, Phys. Fluids 23, 1205 (1980).

[39] E. A. Frieman and L. Chen, Phys. Fluids B 25, 502 (1982).

[40] H. Biglari and L. Chen, Phys. Rev. Lett. 67, 3681 (1991).

[41] S. Briguglio, G. Vlad, F. Zonca, and C. Kar, Phys. Plasmas 2, 3711 (1995).

[42] J. Zhu, G. Y. Fu, and Z. W. Ma, Phys. Plasmas 20, 072508 (2013).

[43] O. P. Pogutse and E. I. Yurchenko, JETP Lett. 28, 318 (1978).

[44] P. Andersson and J. Weiland, Phys. Fluids 29, 1744 (1986).

[45] F. Zonca, P. Buratti, A. Cardinali, L. Chen, J.-Q. Dong, Y.-X. Long, A. Milovanov, F. Romanelli, P. Smeulders, L. Wang, Z.-T. Wang, C. Castaldo, R. Cesario, E. Giovannozzi, M. Marinucci, and V. P. Ridolfini, Nucl. Fusion 47, 1588 (2007).

[46] F. Zonca, L. Chen, A. Botrugno, P. Buratti, A. Cardinali, R. Cesario, V. P. Ridolfini1, and the JET-EFDA contributors, Nucl. Fusion 49, 085009 (2009). 